\title{
HEAT FLOW THROUGH A THIN COOLED PIPE FILLED WITH A MICROPOLAR FLUID
}

\author{
Michal Beneř \\ Czech Technical University, Faculty of Civil Engineering, Czech Republic \\ IGOR PAŽANIN \\ University of Zagreb, Faculty of Science, Zagreb, Croatia; e-mail address: pazanin@math.hr \\ Francisco JaVier SuÁrez-Grau \\ Universidad de Sevilla, Facultad de Matemáticas, Sevilla, Spain
}

\begin{abstract}
In this paper, a non-isothermal flow of a micropolar fluid in a thin pipe with circular cross-section is considered. The fluid in the pipe is cooled by the exterior medium and the heat exchange on the lateral part of the boundary is described by Newton's cooling condition. Assuming that the hydrodynamic part of the system is provided, we seek for the micropolar effects on the heat flow using the standard perturbation technique. Different asymptotic models are deduced depending on the magnitude of the Reynolds number with respect to the pipe thickness. The critical case is identified and the explicit approximation for the fluid temperature is built improving the known result for the classical Newtonian flow as well. The obtained results are illustrated by some numerical simulations.
\end{abstract}

Keywords: pipe flow, heat conduction, micropolar fluid, asymptotic analysis

\section{Introduction}

The Navier-Stokes model of classical hydrodynamics is based on the assumption that the fluid particles do not posses any internal structure. However, in the case of fluids whose particles have complex shapes (e.g. polymeric suspensions, liquid crystals, muddy fluids, animal blood, even water in models with small scales), fluid particles can exhibit some microscopical effects such as rotation and shrinking. For such fluids, the local structure and micro-motions of the fluid elements cannot be ignored and one of the best-established theories covering those phenomenae is the micropolar fluid theory, introduced by Eringen (1966). Physically, micropolar fluids consist of rigid, spherical particles suspended in a viscous medium where the deformation of the particles is ignored. The individual particles may rotate (independently of the movement of the fluid) and, thus, a new vector field, the angular velocity field of rotation of particles (microrotation) is introduced to classical pressure and velocity fields. Correspondingly, one new vector equation is added expressing the conservation of the angular momentum. As a result, a non-Newtonian model is obtained representing an important generalization of the Navier-Stokes equations. As such, it describes the behavior of numerous real fluids better than the classical model, especially when the characteristic dimensions of the flow (e.g. diameter of the pipe) become very small.

Here we investigate the non-isothermal 3D flow of a micropolar fluid in a thin (or long) cylindrical pipe. The problem is described by a complex nonlinear system of PDEs in which micropolar equations are coupled with the heat conduction equation (see Lukaszewicz, 1999). The full coupled system is very difficult to be handled, especially if one wants to analytically construct an asymptotic approximation of the flow. Therefore, in this paper, we are going to consider only the heat flow in a thin pipe assuming that the velocity distribution is known. That means that the governing problem is being described by the non-stationary heat equation 
with given velocity in the convection term. In view of the applications we want to model (heat exchangers, pipelines, etc.), we assume that the pipe is plunged in an ambient medium whose temperature is different from the fluid temperature. The heat exchange between the fluid and surrounding medium is being described by Robin's boundary condition resulting from Newton's cooling law. Our goal is to derive a simplified mathematical model describing the asymptotic behavior of the fluid temperature in such a situation.

Seeking primarily for the micropolar effects, one needs to focus on the convection term carrying the effects of the fluid microstructure in the velocity distribution. Following Marušic et al. (2008), the idea is to assume that the Reynolds number may depend on the small parameter $\varepsilon$ (being the ratio between pipe thickness and its length) and to deduce various models depending on its magnitude with respect to $\varepsilon$. In the mentioned paper, classical Newtonian flow has been treated and the critical case is identified in which the effects of the convection term and the surrounding temperature are of the same order. Avoiding computation of the formal asymptotic expansion, the method employed by Marušić et al. (2008) is very elegant but, unfortunately, cannot be employed in the micropolar setting. The reason lies in the fact that the approximation for velocity feels the microstructure effects in its corrector (see Appendix). In view of that, we are forced to change the methodology and try to formally derive a higher-order asymptotic approximation for the fluid temperature acknowledging the effects we seek for. Starting from the non-dimensional setting and using the two-scale asymptotic technique, we manage to construct an explicit approximation in the critical case clearly showing in which way the fluid microstructure and exterior temperature affect the heat flow inside the pipe. This is especially important with regard to numerical computations.

The isothermal flow of a micropolar fluid was successfully considered both in 2D, see Dupuy et al. $(2004,2008)$ and in a more realistic 3D case, see Pažanin (2011a,b). Taking into account the thermal effects as well, non-isothermal flows have gained much attention in the recent years, see e.g. Prathap Kumar et al. (2010), Si et al. (2013), Ali and Ashraf (2014). To our knowledge, so far only simplified 2D setting (in which the microrotation is a scalar function) has been studied and the influence of the surrounding medium has been neglected in the process. For that reason, in the present paper we address the $3 \mathrm{D}$ problem in a cooled pipe describing a real-life situation.

\section{Position of the problem}

Let $B=B(0,1) \subset \mathbb{R}^{2}$ be a unit circle. We study the flow in a straight pipe with length $L$ and cross-section diameter $d$ given by $\widehat{\Omega}=\left\{\widehat{x}=\left(\widehat{x}_{1}, \widehat{x}_{2}, \widehat{x}_{3}\right) \in \mathbb{R}^{3}: 0<\widehat{x}_{1}<L,\left(\widehat{x}_{2}, \widehat{x}_{3}\right) \in d B\right\}$. We take that the ratio $\varepsilon=d / L$ is small meaning that we consider the problem in a cylindrical pipe which is either very thin or very long. It is supposed that the pipe is filled with an incompressible micropolar fluid. Motivated by the framework of heat exchangers, we assume that the fluid inside the pipe is cooled by the exterior medium so we prescribe Newton's cooling condition on the pipe lateral boundary. As explained in Introduction, our intention is to study the thermodynamic part of the system assuming that the velocity distribution is known and given by the approximation provided in Appendix.

In such a situation, it is plausible to work with the problem written in a non-dimensional form. In view of that, we introduce the non-dimensional variable $x=\left(x_{1}, x_{2}, x_{3}\right)=\widehat{x} / L$ and, correspondingly, the domain $\Omega^{\varepsilon}=(0,1) \times \varepsilon B$. We denote by $\Gamma^{\varepsilon}=(0,1) \times \varepsilon \partial B$ the lateral boundary of the pipe. All physical properties of the fluid are assumed to be constant: $\nu$ - kinematic Newtonian viscosity, $\kappa$ - thermal conductivity and $c_{p}$ - specific heat capacity at constant pressure. We set density of the fluid to be equal to one, for the sake of notational simplicity. In the non-dimensional framework, three characteristic numbers appear: $\operatorname{Re}^{\varepsilon}=\nu^{-1} U_{0} L$ - Reynolds number, $\operatorname{Pr}=\kappa^{-1} \nu c_{p}$ - Prandtl number and $\mathrm{Nu}=\beta L \kappa^{-1}-$ Nusselt number. Here $U_{0}$ denotes the 
characteristic velocity of the fluid, while $\beta$ stands for the heat transfer coefficient coming from Newton's cooling condition. We take the characteristic time of the process as $T_{0}=L^{2} c_{p} \kappa^{-1}$ and, correspondingly, introduce rescaled time as $t=\tau / T_{0}$. As a result, our problem for the unknown fluid temperature $\Phi^{\varepsilon}(x, t)$ can be written as follows

$$
\begin{aligned}
& \frac{\partial \Phi^{\varepsilon}}{\partial t}-\Delta \Phi^{\varepsilon}+\operatorname{Re}^{\varepsilon} \operatorname{Prv}^{\varepsilon} \cdot \nabla \Phi^{\varepsilon}=0 \quad \text { in } \quad \Omega^{\varepsilon} \times(0, T) \\
& \Phi^{\varepsilon}=\theta_{k} \quad \text { for } \quad x_{1}=k \quad(k=0,1) \\
& \frac{\partial \Phi^{\varepsilon}}{\partial \mathbf{n}}=\operatorname{Nu}\left(G-\Phi^{\varepsilon}\right) \quad \text { on } \quad \Gamma^{\varepsilon} \times(0, T) \quad \Phi^{\varepsilon}(x, 0)=\Phi^{0}(x) \quad x \in \Omega^{\varepsilon}
\end{aligned}
$$

The fluid velocity enters the above system as the known function $\mathbf{v}^{\varepsilon}\left(x_{1}, x_{2}, x_{3}\right)=$ $\mathbf{u}^{\varepsilon}\left(x_{1}, x_{2} / \varepsilon, x_{3} / \varepsilon\right)$ with $\mathbf{u}^{\varepsilon}$ being provided in Appendix. Robin's boundary condition $(2.2)_{1}$ models the heat exchange between the fluid inside the pipe and surrounding medium (n denotes exterior unit normal on $\Gamma^{\varepsilon}$ ). To simplify the calculations a little bit, exterior temperature $G$ and boundary temperatures $\theta_{k}$ are assumed to be independent of cross-section variables, i.e. $G=G\left(x_{1}, t\right)$ and $\theta_{k}=\theta_{k}(t)(k=0,1)$.

This is the formal setting of our problem. It consists of (linear) convection-diffusion equation equipped with the appropriate mixed boundary conditions for temperature. The existence and uniqueness issues for such a problem are resolved and well-known (see e.g. Ladyzhenskaya et al., 1967) and, thus, are not going to be discussed here. Using asymptotic analysis with respect to $\varepsilon$, we want to derive a macroscopic law describing the behavior of fluid temperature clearly acknowledging the effects of the fluid microstructure and surrounding temperature.

\section{Asymptotic analysis}

Denoting $x^{\prime}=\left(x_{2}, x_{3}\right)$, we introduce the fast variable $y^{\prime}=\left(y_{2}, y_{3}\right)$ as $y^{\prime}=x^{\prime} / \varepsilon$. Correspondingly, we introduce the new unknown function $\varphi^{\varepsilon}\left(x_{1}, y^{\prime}, t\right)=\Phi^{\varepsilon}\left(x_{1}, \varepsilon y^{\prime}, t\right)$. Taking into account that $\mathbf{u}^{\varepsilon}\left(x_{1}, y^{\prime}\right)=\mathbf{u}_{0}\left(y^{\prime}\right)+\varepsilon \mathbf{u}_{1}\left(x_{1}, y^{\prime}\right)$ (see Appendix), $\varphi^{\varepsilon}$ satisfies the following (rescaled) equation

$$
\frac{\partial \varphi^{\varepsilon}}{\partial t}-\frac{1}{\varepsilon^{2}} \Delta_{y^{\prime}} \varphi^{\varepsilon}-\frac{\partial^{2} \varphi^{\varepsilon}}{\partial x_{1}^{2}}+\operatorname{PrRe}^{\varepsilon}\left(u_{0}^{1} \frac{\partial \varphi^{\varepsilon}}{\partial x_{1}}+u_{1}^{2} \frac{\partial \varphi^{\varepsilon}}{\partial y_{2}}+u_{1}^{3} \frac{\partial \varphi^{\varepsilon}}{\partial y_{3}}+\varepsilon u_{1}^{1} \frac{\partial \varphi^{\varepsilon}}{\partial x_{1}}\right)=0
$$

The above equation is posed in $\Omega \times(0, T)$, where $\Omega=(0,1) \times B$. Here and in the sequel, we denote

$$
\Delta_{y^{\prime}} \phi=\frac{\partial^{2} \phi}{\partial y_{2}^{2}}+\frac{\partial^{2} \phi}{\partial y_{3}^{2}} \quad \nabla_{y^{\prime}} \phi=\frac{\partial \phi}{\partial y_{2}} \mathbf{e}_{2}+\frac{\partial \phi}{\partial y_{3}} \mathbf{e}_{3}
$$

for a scalar function $\phi$ and Cartesian basis $\left(\mathbf{e}_{1}, \mathbf{e}_{2}, \mathbf{e}_{3}\right)$. Newton's cooling condition $(2.2)_{1}$ after rescaling has the following form

$$
\nabla_{y^{\prime}} \varphi^{\varepsilon} \cdot y^{\prime}=\varepsilon \mathrm{Nu}\left(G-\varphi^{\varepsilon}\right) \quad \text { on } \quad \Gamma \times(0, T)
$$

We express the unknown temperature $\varphi^{\varepsilon}$ as an asymptotic expansion in powers of the small parameter $\varepsilon$

$$
\varphi^{\varepsilon}\left(x_{1}, y^{\prime}, t\right)=\varphi_{0}\left(x_{1}, y^{\prime}, t\right)+\varepsilon \varphi_{1}\left(x_{1}, y^{\prime}, t\right)+\varepsilon^{2} \varphi_{2}\left(x_{1}, y^{\prime}, t\right)+\ldots
$$

and substitute it in (3.1) and (3.2). It is essential to observe the following: if we kept the Reynolds number $\operatorname{Re}^{\varepsilon}$ constant (independent of $\varepsilon$ ), then we would obtain no contribution of the convection term in the macroscopic model. In fact, the exterior temperature $G$ would completely dominate 
the process and no effects of the fluid microstructure could be observed (see a) below). Thus, we adopt the idea from Marušić et al. (2008) and use the fact that $\operatorname{Re}^{\varepsilon}$ can be compared with the small parameter $\varepsilon$ in a various way leading to possible different asymptotic models depending on its order of magnitude. Indeed, plugging (3.3) in (3.1) and (3.2), after collecting equal powers of $\varepsilon$, we detect two characteristic cases:

1. $\operatorname{Re}^{\varepsilon} \ll \mathcal{O}\left(\frac{1}{\varepsilon}\right)$

The lowest order approximation satisfies the following problem

$\frac{1}{\varepsilon^{2}}: \Delta_{y^{\prime}} \varphi_{0}=0 \quad$ in $\quad B \quad 1: \nabla_{y^{\prime}} \varphi_{0} \cdot y^{\prime}=0 \quad$ on $\quad \partial B, \quad\left(x_{1}, t\right) \in(0,1) \times(0, T)$

providing $\varphi_{0}=\varphi_{0}\left(x_{1}, t\right)$. The next term in the expansion is given by

$$
\frac{1}{\varepsilon}: \Delta_{y^{\prime}} \varphi_{1}=0 \quad \text { in } \quad B \quad 1: \nabla_{y^{\prime}} \varphi_{1} \cdot y^{\prime}=\mathrm{Nu}\left(G-\varphi_{0}\right) \quad \text { on } \quad \partial B
$$

For fixed $\left(x_{1}, t\right) \in(0,1) \times(0, T)$, this is the standard Neumann problem for the Laplace equation. Thus, it will be solvable if $\int_{\partial B} \mathrm{Nu}\left(G-\varphi_{0}\right) d y^{\prime}=0$ implying $\varphi_{0}=G$. It means that, for $\operatorname{Re}^{\varepsilon} \ll \mathcal{O}(1 / \varepsilon)$, the fluid inside the pipe assumes temperature of the surrounding medium and that the effects of the boundary temperature $\theta_{k}$ are negligible. Moreover, the micropolar effects do not appear even in higher-order terms.

2. $\operatorname{Re}^{\varepsilon} \gg \mathcal{O}\left(\frac{1}{\varepsilon}\right)$

In this case, the convection term becomes dominant, but $\nabla_{y^{\prime}} \varphi_{0} \cdot y^{\prime}=0$ on $\partial B$ (see (3.2)). Therefore, we conclude that the effects of the surrounding temperature $G$ would be negligible on this assumption.

Taking into account the above discussion, we conclude that the most interesting case is when $\operatorname{Re}^{\varepsilon}=\mathcal{O}(1 / \varepsilon)$ since it will lead to the asymptotic model in which all the effects we seek for are balanced. Thus, in the sequel, we perform careful analysis in this critical case.

\subsection{Critical case $\operatorname{Re}^{\varepsilon}=\mathcal{O}\left(\frac{1}{\varepsilon}\right)$}

To simplify the notation let us take $\operatorname{Re}^{\varepsilon}=1 / \varepsilon$. The zero order term in the asymptotic expansion is described by problem (3.4) so, as above, we conclude $\varphi_{0}=\varphi_{0}\left(x_{1}, t\right)$. However, the next term is given by

$$
\frac{1}{\varepsilon}: \quad \Delta_{y^{\prime}} \varphi_{1}=\operatorname{Pr} u_{0}^{1} \frac{\partial \varphi_{0}}{\partial x_{1}} \quad \text { in } \quad B \quad \varepsilon: \nabla_{y^{\prime}} \varphi_{1} \cdot y^{\prime}=\mathrm{Nu}\left(G-\varphi_{0}\right) \quad \text { on } \quad \partial B
$$

Taking into account that $u_{0}^{1}=2 Q\left(1-\left|y^{\prime}\right|^{2}\right)$ (see Appendix), the compatibility condition for the existence of the solution to problem (3.5) gives

$$
\operatorname{Pr} Q \frac{\partial \varphi_{0}}{\partial x_{1}}-2 \mathrm{Nu}\left(G-\varphi_{0}\right)=0 \quad \text { in } \quad(0,1) \times(0, T)
$$

For fixed $t \in(0, T)$, this is in fact, a linear ODE for $\varphi_{0}$ (with respect to $x_{1}$ ) which can be easily solved. To assure uniqueness, we need one boundary condition and the natural choice is $\varphi_{0}(0, t)=\theta_{0}$. Such a choice of the boundary condition is obvious from the physical point of view (the temperature of the fluid exiting the pipe should not be prescribed in advance) and has been 
rigorously justified in the case of Newtonian flow, see Marušić et al. (2008). Consequently, we obtain

$$
\varphi_{0}\left(x_{1}, t\right)=\mathrm{e}^{-\frac{2 \mathrm{Nu}}{\operatorname{Pr} Q} x_{1}}\left(\theta_{0}(t)+\frac{2 \mathrm{Nu}}{\operatorname{Pr} Q} \int_{0}^{x_{1}} \mathrm{e}^{\frac{2 \mathrm{Nu}}{\operatorname{Pr} Q}} G(\xi, t) d \xi\right)
$$

In view of (3.6), the problem for $\varphi_{1}$ becomes

$$
\begin{aligned}
& \Delta_{y^{\prime}} \varphi_{1}=4 \mathrm{Nu}\left(G-\varphi_{0}\right)\left(1-\left|y^{\prime}\right|^{2}\right) \quad \text { in } B \\
& \nabla_{y^{\prime}} \varphi_{1} \cdot y^{\prime}=\mathrm{Nu}\left(G-\varphi_{0}\right) \quad \text { on } \partial B
\end{aligned}
$$

It can be explicitly solved by passing to polar coordinates, leading to

$$
\varphi_{1}\left(x_{1}, y^{\prime}, t\right)=\mathrm{Nu}\left(G-\varphi_{0}\right)\left(\left|y^{\prime}\right|^{2}-\frac{1}{4}\left|y^{\prime}\right|^{4}\right)+C\left(x_{1}, t\right)
$$

Here $C\left(x_{1}, t\right)$ is a function to be determined after identifying the problem for $\varphi_{2}$

$$
\begin{aligned}
& 1: \quad \frac{\partial \varphi_{0}}{\partial t}-\Delta_{y^{\prime}} \varphi_{2}-\frac{\partial^{2} \varphi_{0}}{\partial x_{1}^{2}}+\operatorname{Pr}\left(u_{0}^{1} \frac{\partial \varphi_{1}}{\partial x_{1}}+u_{1}^{2} \frac{\partial \varphi_{1}}{\partial y_{2}}+u_{1}^{3} \frac{\partial \varphi_{1}}{\partial y_{3}}+u_{1}^{1} \frac{\partial \varphi_{0}}{\partial x_{1}}\right)=0 \quad \text { in } \quad B \\
& \varepsilon^{2}: \nabla_{y^{\prime}} \varphi_{2} \cdot y^{\prime}=-\mathrm{Nu} \varphi_{1} \quad \text { on } \quad \partial B, \quad\left(x_{1}, t\right) \in(0,1) \times(0, T)
\end{aligned}
$$

The above system will be solvable if and only if

$$
\begin{aligned}
& \pi \frac{\partial \varphi_{0}}{\partial t}-\pi \frac{\partial^{2} \varphi_{0}}{\partial x_{1}^{2}}+\operatorname{Pr} \int_{B} u_{0}^{1} \frac{\partial \varphi_{1}}{\partial x_{1}} d y^{\prime}+\operatorname{Pr} \int_{B} u_{1}^{2} \frac{\partial \varphi_{1}}{\partial y_{2}} d y^{\prime}+\operatorname{Pr} \int_{B} u_{1}^{3} \frac{\partial \varphi_{1}}{\partial y_{3}} d y^{\prime} \\
& +\operatorname{Pr} \frac{\partial \varphi_{0}}{\partial x_{1}} \int_{B} u_{1}^{1} d y^{\prime}=-\mathrm{Nu} \int_{\left|y^{\prime}\right|=1} \varphi_{1} d y^{\prime}
\end{aligned}
$$

The components of the velocity corrector $\mathbf{u}_{1}$ appearing in $(3.10)_{1}$ have the following form (see Appendix)

$$
\begin{aligned}
& u_{1}^{1}\left(x_{1}, y^{\prime}\right)=\frac{1}{8}\left(\left|y^{\prime}\right|^{2}-1\right)\left[\left(\frac{d f_{2}}{d x_{1}}+2 N^{2} \frac{g_{3}}{2 R_{1}+R_{2}}\right) y_{2}+\left(\frac{d f_{3}}{d x_{1}}-2 N^{2} \frac{g_{2}}{2 R_{1}+R_{2}}\right) y_{3}\right] \\
& u_{1}^{2}\left(x_{1}, y^{\prime}\right)=-\frac{N^{2}}{8 R_{1}} g_{1}\left(x_{1}\right)\left(1-\left|y^{\prime}\right|^{2}\right) y_{3} \\
& u_{1}^{3}\left(x_{1}, y^{\prime}\right)=\frac{N^{2}}{8 R_{1}} g_{1}\left(x_{1}\right)\left(1-\left|y^{\prime}\right|^{2}\right) y_{2}
\end{aligned}
$$

Taking into account expression (3.9), by direct integration we obtain that the last three integrals on the left-hand side in (3.11) are equal to zero. Thus, from (3.11) we deduce an equation for $C\left(x_{1}, t\right)$

$$
\begin{aligned}
& \operatorname{Pr} Q \frac{\partial C}{\partial x_{1}}+2 \mathrm{Nu} C=D \\
& D\left(x_{1}, t\right)=\frac{\partial \varphi_{0}}{\partial t}-\frac{\partial^{2} \varphi_{0}}{\partial x_{1}^{2}}+\frac{7 Q \mathrm{NuPr}}{24}\left(\frac{\partial \varphi_{0}}{\partial x_{1}}-\frac{\partial G}{\partial x_{1}}\right)+\frac{3}{2} \mathrm{Nu}^{2}\left(\varphi_{0}-G\right)
\end{aligned}
$$

Endowing it with the boundary condition $C(0, t)=0$, we get

$$
C\left(x_{1}, t\right)=\frac{1}{\operatorname{Pr} Q} \mathrm{e}^{-\frac{2 \mathrm{Nu}}{\operatorname{Pr} Q} x_{1}} \int_{0}^{x_{1}} \mathrm{e}^{\frac{2 \mathrm{Nu}}{\operatorname{Pr} Q}} D(\xi, t) d \xi .
$$


Remark 1. The corrector $\varphi_{1}$ is computed to satisfy equation $(3.8)_{1}$ and cooling condition $(3.8)_{2}$. Note that the boundary condition at $x_{1}=0$ could not be taken into account in the process. As a consequence, $\varphi_{1}\left(0, y^{\prime}, t\right) \neq 0$ implying $\varphi_{0}+\varepsilon \varphi_{1} \neq \theta_{0}$, i.e. the boundary temperature at the inlet is being modified by the higher-order term. We can fix that by introducing the appropriate boundary layer corrector depending on the dilated variable $\left(y_{1}, y^{\prime}\right)=$ $\left(x_{1} / \varepsilon, x^{\prime} / \varepsilon\right)$, see e.g. Marušić-Paloka and Pažanin (2009). However, such a corrector would have an exponential decay towards zero (as $y_{1} \rightarrow+\infty$ ) meaning that it would not affect the approximation outside the boundary layer (it would only serve for the convergence proof which is out of the scope of the present paper). Thus, there is no reason to formally correct the approximation $\varphi_{0}+\varepsilon \varphi_{1}$ in the vicinity of $x_{1}=0$ since the effects of such correction would not contribute to the macroscopic model except in the small boundary layer near the pipe inlet.

The corrector $\varphi_{1}$ is given in an explicit form by (3.9) and (3.12). We observe that there are contributions of surrounding and boundary temperature, but we find no micropolar effects at this order. Therefore, we have to continue computation and try to construct a higher-order corrector $\varphi_{2}$. Taking into account (3.7) and (3.9) and the expression for the velocity distribution, problem (3.10) can be written as

$$
\begin{aligned}
& \Delta_{y^{\prime}} \varphi_{2}=\left[-\frac{\partial \varphi_{0}}{\partial t}+\frac{2 \mathrm{Nu}}{\operatorname{Pr} Q} \frac{\partial G}{\partial x_{1}}+\left(\frac{2 \mathrm{Nu}}{\operatorname{Pr} Q}\right)^{2}\left(\varphi_{0}-G\right)\right]\left(1-2\left|y^{\prime}\right|^{2}\right)-4 \mathrm{Nu} C\left(1-\left|y^{\prime}\right|^{2}\right) \\
& -\mathrm{Nu}^{2}\left(G-\varphi_{0}\right)\left(1-\left|y^{\prime}\right|^{2}\right)\left(\frac{11}{6}+4\left|y^{\prime}\right|^{2}-\left|y^{\prime}\right|^{4}\right) \\
& \quad+\frac{1}{2} \operatorname{PrNu} Q \frac{\partial G}{\partial x_{1}}\left(1-\left|y^{\prime}\right|^{2}\right)\left(-\frac{7}{6}+4\left|y^{\prime}\right|^{2}-\left|y^{\prime}\right|^{4}\right) \\
& \quad+\frac{\mathrm{Nu}}{4 Q}\left(\varphi_{0}-G\right)\left(1-\left|y^{\prime}\right|^{2}\right) H_{1} y_{2}+\frac{\mathrm{Nu}}{4 Q}\left(\varphi_{0}-G\right)\left(1-\left|y^{\prime}\right|^{2}\right) H_{2} y_{3} \quad \text { in } B \\
& \nabla_{y^{\prime}} \varphi_{2} \cdot y^{\prime}=-\frac{3}{4} \mathrm{Nu}^{2}\left(G-\varphi_{0}\right)-\mathrm{Nu} C \quad \text { on } \quad \partial B, \quad\left(x_{1}, t\right) \in(0,1) \times(0, T)
\end{aligned}
$$

Here we denote

$$
H_{1}\left(x_{1}\right)=\frac{d f_{2}}{d x_{1}}+2 N^{2} \frac{g_{3}}{2 R_{1}+R_{2}} \quad H_{2}\left(x_{1}\right)=\frac{d f_{3}}{d x_{1}}-2 N^{2} \frac{g_{2}}{2 R_{1}+R_{2}}
$$

coming from $u_{1}^{1}$. Problem (3.13) can be analytically solved by introducing six auxiliary problems corresponding to each term on the right-hand side of $(3.13)_{1}$ and passing to polar coordinates. We leave the reader to confirm that

$$
\begin{aligned}
& \varphi_{2}\left(x_{1}, y^{\prime}, t\right)=\left[-\frac{\partial \varphi_{0}}{\partial t}+\frac{2 \mathrm{Nu}}{\operatorname{Pr} Q} \frac{\partial G}{\partial x_{1}}+\left(\frac{2 \mathrm{Nu}}{\operatorname{Pr} Q}\right)^{2}\left(\varphi_{0}-G\right)\right]\left(\frac{1}{4}\left|y^{\prime}\right|^{2}-\frac{1}{8}\left|y^{\prime}\right|^{4}\right) \\
& -\mathrm{Nu} C\left(\left|y^{\prime}\right|^{2}-\frac{1}{4}\left|y^{\prime}\right|^{4}\right)-\mathrm{Nu}^{2}\left(G-\varphi_{0}\right)\left(\frac{11}{24}\left|y^{\prime}\right|^{2}+\frac{13}{96}\left|y^{\prime}\right|^{4}-\frac{5}{36}\left|y^{\prime}\right|^{6}+\frac{1}{64}\left|y^{\prime}\right|^{8}\right) \\
& +\frac{1}{2} \operatorname{PrNu} Q \frac{\partial G}{\partial x_{1}}\left(-\frac{7}{24}\left|y^{\prime}\right|^{2}+\frac{31}{96}\left|y^{\prime}\right|^{4}-\frac{5}{36}\left|y^{\prime}\right|^{6}+\frac{1}{64}\left|y^{\prime}\right|^{8}\right) \\
& +\frac{\mathrm{Nu}}{4 Q}\left(G-\varphi_{0}\right)\left(\frac{d f_{2}}{d x_{1}}+\frac{2 N^{2} g_{3}}{2 R_{1}+R_{2}}\right)\left(\frac{1}{6}-\frac{1}{8}\left|y^{\prime}\right|^{2}+\frac{1}{24}\left|y^{\prime}\right|^{4}\right) y_{2} \\
& +\frac{\mathrm{Nu}}{4 Q}\left(G-\varphi_{0}\right)\left(\frac{d f_{3}}{d x_{1}}-\frac{2 N^{2} g_{2}}{2 R_{1}+R_{2}}\right)\left(\frac{1}{6}-\frac{1}{8}\left|y^{\prime}\right|^{2}+\frac{1}{24}\left|y^{\prime}\right|^{4}\right) y_{3}
\end{aligned}
$$

This is the end of the formal derivation. Our asymptotic solution has the following form

$$
\begin{aligned}
& \varphi_{\text {approx }}^{\varepsilon}\left(x_{1}, y^{\prime}, t\right)=\varphi_{0}\left(x_{1}, t\right)+\varepsilon \varphi_{1}\left(x_{1}, y^{\prime}, t\right)+\varepsilon^{2} \varphi_{2}\left(x_{1}, y^{\prime}, t\right) \\
& \left(x_{1}, y^{\prime}\right) \in \Omega \quad t \in(0, T)
\end{aligned}
$$


Since the functions $\varphi_{i}(i=0,1,2)$ are all given in the explicit form, see (3.7), (3.9), (3.14), we can easily detect all the effects we were originally interested for. The first part of the solution, namely $\varphi_{0}+\varepsilon \varphi_{1}$, is influenced by the effects of cooling and upstream boundary temperature, and it can be viewed as an improvement of the result by Marušić et al. (2008) for the classical Newtonian flow. No effects of the fluid microstructure can be seen there. However, in the second-order corrector $\varphi_{2}$ we can clearly observe the correction coming due to the non-Newtonian (micropolar) nature of the fluid. The correction, i.e. the difference between $\varphi_{0}+\varepsilon \varphi_{1}$ and $\varphi_{\text {approx }}^{\varepsilon}$ can be clearly observed in the numerical examples in the following Section as well.

\section{Numerical examples}

In this Section we aim to visually compare the function $\varphi_{0}+\varepsilon \varphi_{1}$ (representing the approximation for the Newtonian flow) with the asymptotic approximation $\varphi_{a p p r o x}^{\varepsilon}$ for the generalized micropolar flow. It must be emphasized that there is very little information in the existing literature concerning the values of micropolar parameters. We use the reference by Papautsky et al. (1999) where $\nu=2.9 \cdot 10^{-3}, \nu_{r}=2.32 \cdot 10^{-4}, c_{a}+c_{d}=10^{-6}$ are reported for the micropolar viscosity constants. Furthermore, we neglect the body force $\mathbf{f}$, while $\mathbf{g}$ is taken to be a point couple, see e.g. Shu and Lee (2008) (sequence $\delta_{n}=n /\left[\pi\left(1+n^{2} x_{1}^{2}\right)\right]$ is employed to approach the sifting property of the Dirac delta function). Exterior and boundary temperatures are assumed to be constant $\left(G=10, \theta_{0}=35\right)$, while for the remaining constants we take $\mathrm{Nu}=3.66, \operatorname{Pr}=4.8$ and $Q=81$. Polar coordinates $\left(\left|y^{\prime}\right|, \vartheta\right)$ are used to describe the domain cross-section $B$, and $2 \mathrm{D}$ comparisons for different magnitudes of the small parameter $\varepsilon$ are presented (see Figs. 1 and 2).
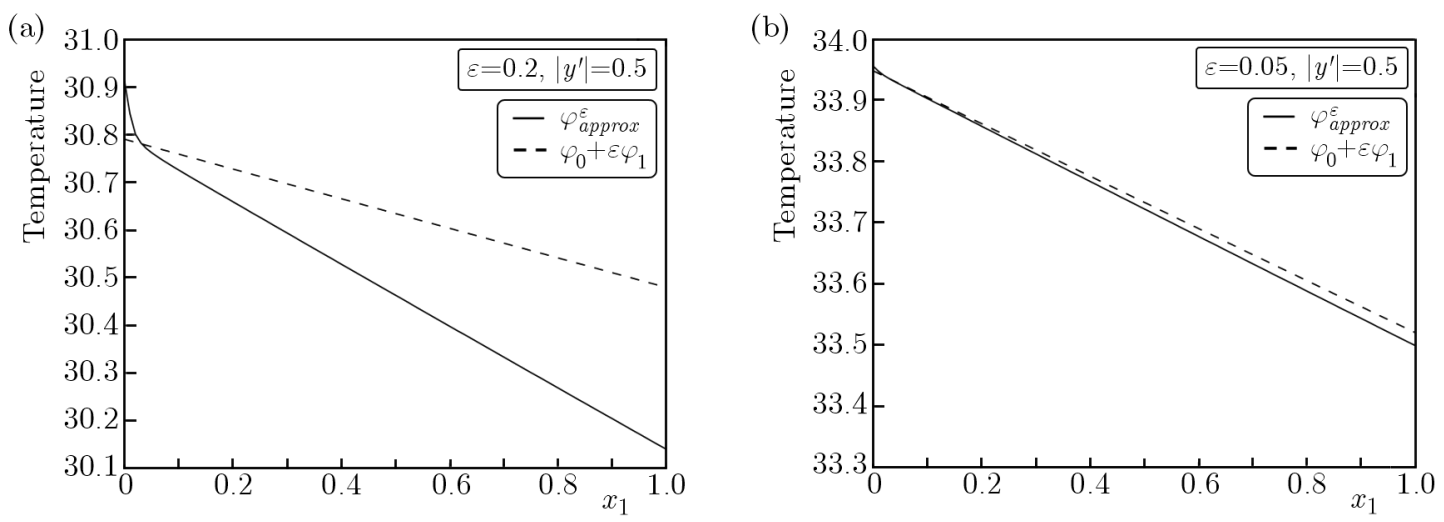

Fig. 1. Temperature distribution for the variable $x_{1}$ for a fixed $\left|y^{\prime}\right|$ and polar angle $\vartheta=\pi / 2$
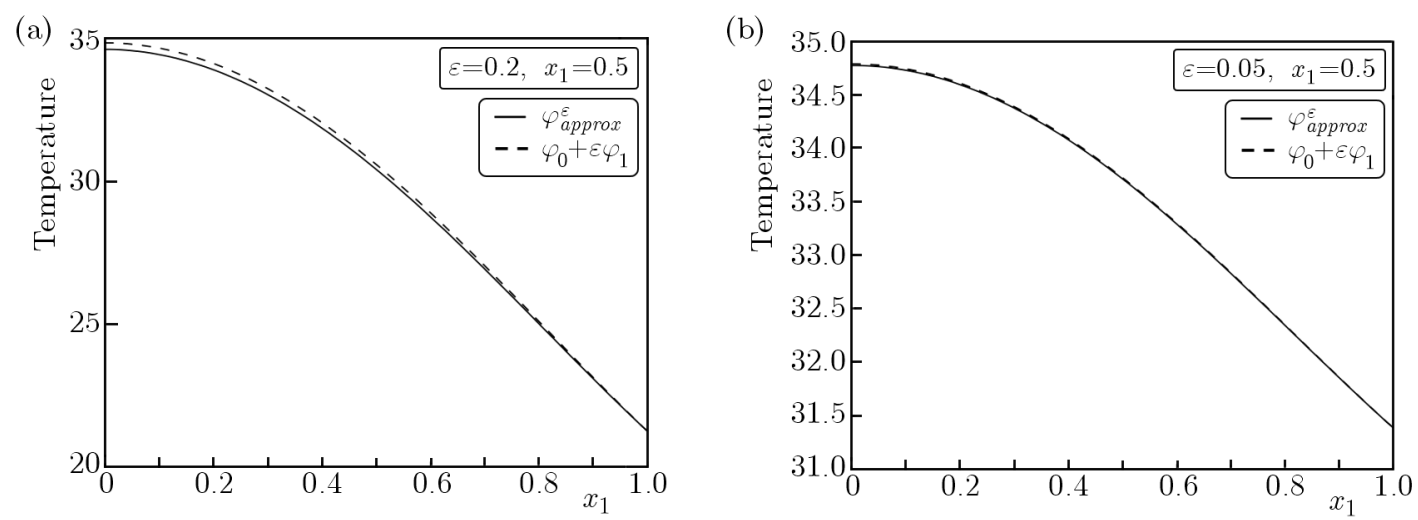

Fig. 2. Temperature distribution for the variable $\left|y^{\prime}\right|$ (with $\vartheta=\pi / 2$ ) and for a fixed $x_{1}$ 


\section{Concluding remarks}

We derived the asymptotic model describing the heat flow through a cylindrical pipe (with thickness $\mathcal{O}(\varepsilon)$ ) in the critical case where the effects of micropolarity, surrounding medium and entering temperature are balanced. The discussion at the beginning of Sec. 3 suggested that the critical case is reached when the Reynolds number is of order $\mathcal{O}\left(\varepsilon^{-1}\right)$. As presented, the correctors in the asymptotic expansion for the fluid temperature can be explicitly computed clearly showing the difference between the classical Newtonian and micropolar flow. It is important to observe that such a more accurate approximation, is particularly interesting if $\varepsilon$ is not too small (e.g. $\varepsilon=0.2$ or $\varepsilon=0.05$ as chosen in the numerical examples). Indeed, the smaller $\varepsilon$ becomes, the difference between the Newtonian and micropolar flow becomes the less obvious (see Figs. 1 and 2). Moreover, by keeping $\varepsilon$ in such a range, we ensure that the critical value of the Reynolds number is not exceeded, i.e. the flow stays in the laminar regime (not becoming turbulent, so we can use the laminar velocity profile from Appendix).

Let us discuss in more details the numerical results obtained in Sec. 4. First, in Fig. 1, we fix the cross-section variable and consider the temperature variations for the variable $x_{1}$. The result clearly suggests that, except in the small boundary layer near the left end of the pipe, the micropolar nature of the fluid enhances the cooling as we move along the pipe. Note that the value of the temperature at $x_{1}=0$ is a bit lower than the imposed $\theta_{0}=35$. That is essentially due to the fact that we have computed the correctors to satisfy the heat equation and the cooling condition, while the boundary condition at the pipe inlet has been neglected. Consequently, a boundary layer appear (in the vicinity of $x_{1}=0$ ) having no influence on the effective flow far from the pipe inlet, as explained in Remark 1. On the other hand, by fixing $x_{1}$, from Fig. 2 we deduce that the micropolar effects enhances the cooling far from the pipe lateral boundary. Indeed, as we approach the boundary, those effects become negligible due to the influence of the surrounding temperature.

To conclude, we believe that the above observations could improve the known engineering practice by acknowledging the obtained micropolar effects on the cooling process.

\section{Appendix A. Approximation for the velocity}

For reader's convenience, here we provide an asymptotic approximation for the fluid velocity used in the main part of the paper. For detailed (formal) derivation, we refer the reader to Pažanin (2013). Rigorous justification can be recovered from Pažanin (2011).

We start from the linearized micropolar equations (see Lukaszewicz, 1999)

$$
\begin{aligned}
& -\left(\nu+\nu_{r}\right) \Delta \widehat{\mathbf{u}}^{\varepsilon}+\nabla \widehat{p}^{\varepsilon}=2 \nu_{r} \operatorname{rot} \widehat{\mathbf{w}}^{\varepsilon}+\widehat{\mathbf{f}} \\
& \operatorname{div} \widehat{\mathbf{u}}^{\varepsilon}=0 \quad \text { in } \widehat{\Omega}^{\varepsilon} \\
& -\left(c_{a}+c_{d}\right) \Delta \widehat{\mathbf{w}}^{\varepsilon}-\left(c_{0}+c_{d}-c_{a}\right) \nabla \operatorname{div} \widehat{\mathbf{w}}^{\varepsilon}+4 \nu_{r} \widehat{\mathbf{w}}^{\varepsilon}=2 \nu_{r} \operatorname{rot} \widehat{\mathbf{u}}^{\varepsilon}+\widehat{\mathbf{g}}
\end{aligned}
$$

The unknowns are: velocity $\widehat{\mathbf{u}}^{\varepsilon}$, pressure $\widehat{p}^{\varepsilon}$ and microrotation $\widehat{\mathbf{w}}^{\varepsilon}$. Positive constants $\nu_{r}, c_{0}, c_{a}, c_{d}$ represent new viscosity coefficients coming from the asymmetry of the stress tensor. Due to the pipe thickness, the body force $\widehat{\mathbf{f}}=\widehat{\mathbf{f}}\left(\widehat{x_{1}}\right)$ and body couple $\widehat{\mathbf{g}}=\widehat{\mathbf{g}}\left(\widehat{x_{1}}\right)$ are assumed to depend only on the variable going along the pipe. We use standard Dirichlet boundary conditions for the velocity and microrotation and prescribe the pressure drop $\widehat{q}_{0}-\widehat{q}_{L}$ between the pipe ends

$$
\begin{array}{lll}
\widehat{\mathbf{u}}^{\varepsilon}=0 & \text { on } \quad \widehat{\Gamma}^{\varepsilon} \quad \mathbf{e}_{1} \times \widehat{\mathbf{u}}^{\varepsilon}=0 \\
\widehat{p}^{\varepsilon}=\widehat{q}_{i} & \text { for } \quad \widehat{x}_{1}=i \quad(i=0, L) \quad \widehat{\mathbf{w}}^{\varepsilon}=0 \quad \text { on } \quad \partial \widehat{\Omega}^{\varepsilon}
\end{array}
$$


Since we need to work in non-dimensional framework, we introduce

$$
\begin{array}{lll}
x_{1}=\frac{\widehat{x}_{1}}{L} \quad y_{2}=\frac{\widehat{x}_{2}}{\varepsilon L} \quad y_{3}=\frac{\widehat{x}_{3}}{\varepsilon L} & \mathbf{u}^{\varepsilon}=\frac{\widehat{\mathbf{u}}^{\varepsilon}}{U_{0}} \quad \mathbf{w}^{\varepsilon}=\frac{L}{U_{0}} \widehat{\mathbf{w}}^{\varepsilon} \\
p^{\varepsilon}=\frac{\varepsilon^{2} L}{U_{0}\left(\nu+\nu_{r}\right)} \widehat{p}^{\varepsilon} \quad \mathbf{f}=\frac{\varepsilon^{2} L^{2}}{U_{0}\left(\nu+\nu_{r}\right)} \widehat{\mathbf{f}} & \mathbf{g}=\frac{\varepsilon^{2} L}{U_{0}\left(\nu+\nu_{r}\right)} \widehat{\mathbf{g}}
\end{array}
$$

When considering micropolar flows, three non-Newtonian characteristic numbers appear

$$
R_{1}=\frac{c_{a}+c_{d}}{\left(\nu+\nu_{r}\right) L^{2}} \quad R_{2}=\frac{c_{0}+c_{d}-c_{a}}{\left(\nu+\nu_{r}\right) L^{2}} \quad N^{2}=\frac{\nu_{r}}{\nu+\nu_{r}}
$$

The parameters $R_{i}$ are related to characteristic length of the microrotation effects, while $N$ characterizes the coupling between the equations for velocity and microrotation. Note that for $N=0$, the above system becomes decoupled and (A.1) ${ }_{1}$ and (A.1) $)_{2}$ reduce to classical Navier-Stokes equations. In view of that, equations (A.1) in the dimensionless form read

$$
\begin{aligned}
& -\Delta_{y^{\prime}} \mathbf{u}^{\varepsilon}-\varepsilon^{2} \frac{\partial^{2} \mathbf{u}^{\varepsilon}}{\partial x_{1}^{2}}+\frac{1}{\varepsilon} \nabla_{y^{\prime}} p^{\varepsilon}+\frac{\partial p^{\varepsilon}}{\partial x_{1}} \mathbf{e}_{1} \\
& \quad=2 N^{2}\left[\varepsilon\left(\frac{\partial w_{3}^{\varepsilon}}{\partial y_{2}}-\frac{\partial w_{2}^{\varepsilon}}{\partial y_{3}}\right) \mathbf{e}_{1}+\varepsilon \operatorname{rot}_{y^{\prime}} w_{1}^{\varepsilon}-\varepsilon^{2} \frac{\partial w_{3}^{\varepsilon}}{\partial x_{1}} \mathbf{e}_{2}+\varepsilon^{2} \frac{\partial w_{2}^{\varepsilon}}{\partial x_{1}} \mathbf{e}_{3}\right]+\mathbf{f} \\
& \operatorname{div}_{y^{\prime}} \mathbf{u}^{\varepsilon}+\varepsilon \frac{\partial u_{1}^{\varepsilon}}{\partial x_{1}}=0 \\
& -R_{1}\left(\Delta_{y^{\prime}} \mathbf{w}^{\varepsilon}+\varepsilon^{2} \frac{\partial^{2} \mathbf{w}^{\varepsilon}}{\partial x_{1}^{2}}\right)-R_{2}\left[\nabla_{y^{\prime}}\left(\operatorname{div}_{y^{\prime}} \mathbf{w}^{\varepsilon}\right)+\varepsilon \frac{\partial}{\partial x_{1}}\left(\operatorname{div}_{y^{\prime}} \mathbf{w}^{\varepsilon}\right) \mathbf{e}_{1}\right. \\
& \left.+\varepsilon \nabla_{y^{\prime}}\left(\frac{\partial w_{1}^{\varepsilon}}{\partial x_{1}}\right)+\varepsilon^{2} \frac{\partial^{2} w_{1}^{\varepsilon}}{\partial x_{1}^{2}} \mathbf{e}_{1}\right]+4 N^{2} \varepsilon^{2} \mathbf{w}^{\varepsilon} \\
& \quad=2 N^{2}\left[\varepsilon\left(\frac{\partial u_{3}^{\varepsilon}}{\partial y_{2}}-\frac{\partial u_{2}^{\varepsilon}}{\partial y_{3}}\right) \mathbf{e}_{1}+\varepsilon \operatorname{rot}_{y^{\prime}} u_{1}^{\varepsilon}-\varepsilon^{2} \frac{\partial u_{3}^{\varepsilon}}{\partial x_{1}} \mathbf{e}_{2}+\varepsilon^{2} \frac{\partial u_{2}^{\varepsilon}}{\partial x_{1}} \mathbf{e}_{3}\right]+\mathbf{g}
\end{aligned}
$$

where

$$
\operatorname{div}_{y^{\prime}} \mathbf{v}=\frac{\partial v_{2}}{\partial y_{2}}+\frac{\partial v_{3}}{\partial y_{3}} \quad \operatorname{rot}_{y^{\prime}} v_{i}=\frac{\partial v_{i}}{\partial y_{3}} \mathbf{e}_{2}-\frac{\partial v_{i}}{\partial y_{2}} \mathbf{e}_{3} \quad v_{i}=\mathbf{v} \cdot \mathbf{e}_{i}
$$

Now we construct the approximation for the fluid velocity $\mathbf{u}^{\varepsilon}$ by plugging the asymptotic expansions

$$
\begin{aligned}
& \mathbf{u}^{\varepsilon}\left(x_{1}, y^{\prime}\right)=\mathbf{u}_{0}\left(x_{1}, y^{\prime}\right)+\varepsilon \mathbf{u}_{1}\left(x_{1}, y^{\prime}\right)+\ldots \\
& \mathbf{w}^{\varepsilon}\left(x_{1}, y^{\prime}\right)=\mathbf{w}_{0}\left(x_{1}, y^{\prime}\right)+\varepsilon \mathbf{w}_{1}\left(x_{1}, y^{\prime}\right)+\ldots \\
& p^{\varepsilon}\left(x_{1}, y^{\prime}\right)=p_{0}\left(x_{1}\right)+\varepsilon p_{1}\left(x_{1}, y^{\prime}\right)+\ldots
\end{aligned}
$$

into system (A.4) and collecting the terms with equal powers of $\varepsilon$. The zero-order approximation turns out to be the standard Poiseuille solution given by

$$
\begin{aligned}
& \mathbf{u}_{0}\left(y^{\prime}\right)=2 Q\left(1-\left|y^{\prime}\right|^{2}\right) \mathbf{e}_{1} \\
& Q=\frac{1}{\pi} \int_{B} u_{0}^{1} d y^{\prime}=\frac{1}{8}\left(q_{0}-q_{1}+\int_{0}^{1} f_{1}(\xi) d \xi\right)
\end{aligned}
$$

Since we observe no effects of the microstructure here, we have to derive a lower-order term $\mathbf{u}_{1}$ from the velocity expansion. Continuing computation, we arrive at 


$$
\begin{aligned}
& u_{1}^{1}\left(x_{1}, y^{\prime}\right)=\frac{1}{8}\left(\left|y^{\prime}\right|^{2}-1\right)\left[\left(\frac{d f_{2}}{d x_{1}}+2 N^{2} \frac{g_{3}}{2 R_{1}+R_{2}}\right) y_{2}+\left(\frac{d f_{3}}{d x_{1}}-2 N^{2} \frac{g_{2}}{2 R_{1}+R_{2}}\right) y_{3}\right] \\
& u_{1}^{2}\left(x_{1}, y^{\prime}\right)=-\frac{N^{2}}{8 R_{1}} g_{1}\left(x_{1}\right)\left(1-\left|y^{\prime}\right|^{2}\right) y_{3} \\
& u_{1}^{3}\left(x_{1}, y^{\prime}\right)=\frac{N^{2}}{8 R_{1}} g_{1}\left(x_{1}\right)\left(1-\left|y^{\prime}\right|^{2}\right) y_{2}
\end{aligned}
$$

Finally, our asymptotic approximation for the fluid velocity reads

$$
\mathbf{u}^{\varepsilon}\left(x_{1}, y^{\prime}\right)=\mathbf{u}_{0}\left(y^{\prime}\right)+\varepsilon \mathbf{u}_{1}\left(x_{1}, y^{\prime}\right) \quad\left(x_{1}, y^{\prime}\right) \in \Omega
$$

\section{Acknowledgements}

The first author of this work has been supported by the project GAČR 13-18652S. The second author has been supported by the Croatian Science Foundation (project 3955: Mathematical modeling and numerical simulations of processes in thin or porous domains). The third author has been supported by the projects MTM2011-24457 of the Ministerio de Economía y Competitividad and FQM309 of the Junta de Andalucía. The authors would like to thank the referee for his/her helpful comments and suggestions.

\section{References}

1. Ali K., Ashraf M., 2014, Numerical simulation of the micropolar fluid flow and heat transfer in a channel with a shrinking and a stationary wall, Journal of Theoretical and Applied Mechanics, $\mathbf{5 2}, 557-569$

2. Dupuy D., Panasenko G., Stavre R., 2004, Asymptotic methods for micropolar fluids in a tube structure, Mathematical Models and Methods in Applied Sciences, 14, 735-758

3. Dupuy D., Panasenko G., Stavre R., 2008, Asymptotic solution for a micropolar flow in a curvilinear channel, Zeitschrift für Angewandte Mathematik und Mechanik, 88, 793-807

4. ERINGen A.C., 1966, Theory of micropolar fluids, Journal of Mathematics and Mechanics, 16, 1 , $1-18$

5. Ladyzhenskaya O.A., Solonnikov V.A., Uraltseva N.N., 1967, Linear and quasilinear equations of parabolic type, Translations of Mathematical Monographs, 23, American Mathematical Society, Providence, R.I.

6. Lukaszewicz G., 1999, Micropolar Fluids: Theory and Applications, Birkhäuser, Boston

7. Marušić S., Marušić-Paloka E., PaŽAnin I., 2008, Effects of strong convection on the cooling process for a long or thin pipe, C.R. Mécanique, 336, 493-499

8. Marušić-Paloka E., PaŽanin I., 2009, Non-isothermal fluid flow through a thin pipe with cooling, Journal of Applied Analysis, 88, 495-515

9. Papautsky I., Brazzle J., Ameel T., Frazier A.B., 1999, Laminar fluid behaviour in microchannels using micropolar fluid theory, Sensors and Actuators A: Physical, 73, 101-108

10. PAŽAnin I., 2011a, Asymptotic behavior of micropolar fluid flow through a curved pipe, Acta Applicandae Mathematicae, 116, 1-25

11. PAŽAnin I., 2011b, Effective flow of micropolar fluid through a thin or long pipe, Mathematical Problems in Engineering, 2011, Article ID 127070, 18 pages

12. PaŽAnin I., 2013, Modeling of solute dispersion in a circular pipe filled with micropolar fluid, Mathematical and Computer Modelling, 57, 2366-2373 
13. Prathap Kumar J., Umavathi J.C., Chamkha A.J., Pop I., 2010, Fully-developed freeconvective flow of micropolar and viscous fluids in a vertical channel, Applied Mathematical Modelling, 34, 1175-1186

14. Si X., Zheng L., Lin P., Zhang X., Zhang Y., 2013, Flow and heat transfer of a micropolar fluid in a porous channel with expanding or contracting walls, International Journal of Heat and Mass Transfer, 67, 885-895

15. Shu J.-J., LEe J.S., 2008, Fundamental solutions for micropolar fluids, Journal of Engineering Mathematics, 61, 69-78

Manuscript received October 16, 2014; accepted for print December 9, 2014 